\title{
Association of patellofemoral morphology and alignment with the radiographic severity of patellofemoral osteoarthritis
}

\author{
Yike Dai, Heyong Yin, Chongyang Xu, Hongrui Zhang, Ai Guo and Naicheng Diao*
}

\begin{abstract}
Background: Risk factors for the severity of patellofemoral osteoarthritis (PFOA) are poorly understood. This research aims to evaluate the association between patellofemoral joint (PFJ) morphology and alignment with the radiographic severity of PFOA.

Methods: A retrospective analysis of $C T$ scan and lateral radiograph data were acquired in patients with PFOA. The radiographic grade of PFOA and tibiofemoral osteoarthritis (TFOA), lateral and medial trochlear inclination angle, sulcus angle, and the Wiberg classification of patella morphology, the congruence angle, patellar tilt angle, and lateral patellar angles, and tibial tubercle trochlear groove distance (TT-TG) and patella height (i.e., CatonDeschamps index) were assessed using CT scans and sagittal radiographs of the knee. All the PFJ morphology and alignment data were divided into quarters, and the relationships between each of these measures and the severity of PFOA were investigated.
\end{abstract}

Results: By studying 150 patients with PFOA, we found a U-shaped relationship between the Caton-Deschamps index and the severity of PFOA $(P<0.001)$. A lower value of sulcus angle and lateral patellar angle, a higher value of congruence angle, and type III patella were associated with more severity of lateral PFOA. Compared with the highest quarter of each measure, the adjusted odds ratios (OR) of the severity of PFOA in the lowest quarter of sulcus angle, lateral patellar angle, and congruence angle; and type I patella was $8.80(p=0.043), 16.51(P<0.001)$, $0.04(P<0.001)$, and $0.18(p=0.048)$ respectively.

Conclusions: Extreme value of patella height, a higher value of lateral patellar displacement and lateral patellar tilt, lower value of sulcus angle, and type III patella were associated with more severity of PFOA.

Keywords: Patellofemoral osteoarthritis, Sulcus angle, Congruence angle, Patellar tilt, Patella height

\section{Background}

Patellofemoral joint osteoarthritis (PFOA) is a type of knee OA, which is one of the most commonly affected compartments. Approximately, 50\% of patients with knee joint osteoarthritis (KOA) do have some degree of PFOA. The prevalence of PFOA appears to be higher in

\footnotetext{
*Correspondence: diaonaicheng@163.com

Department of Orthopedic, Beijing Friendship Hospital, Capital Medical University, No. 95 Yong'an Road, Xicheng, Beijing 100050, People's Republic of China
}

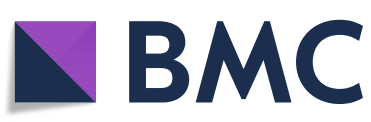

females (age $\geq 50$ years) $(41 \%)$ than in males (age $\geq 50$ years) (23\%) [1]. The most common radiographic findings in patients with knee symptoms were combined tibiofemoral and patellofemoral joint (PFJ) lesions (40\%), followed by isolated PFOA (24\%), isolated tibiofemoral joint osteoarthritis (TFOA) only $4 \%$, and the remaining $32 \%$ had normal radiographic findings [2]. Previously, studies have shown that PFOA most frequently involves the lateral compartment of PFJ [3, 4]. Some studies suggested the risk factors associated with the disease are

(c) The Author(s). 2021 Open Access This article is licensed under a Creative Commons Attribution 4.0 International License, which permits use, sharing, adaptation, distribution and reproduction in any medium or format, as long as you give appropriate credit to the original author(s) and the source, provide a link to the Creative Commons licence, and indicate if changes were made. The images or other third party material in this article are included in the article's Creative Commons. licence, unless indicated otherwise in a credit line to the material. If material is not included in the article's Creative Commons licence and your intended use is not permitted by statutory regulation or exceeds the permitted use, you will need to obtain permission directly from the copyright holder. To view a copy of this licence, visit http://creativecommons.org/licenses/by/4.0/ The Creative Commons Public Domain Dedication waiver (http://creativecommons.org/publicdomain/zero/1.0/) applies to the data made available in this article, unless otherwise stated in a credit line to the data. 
different for PFOA and TFOA [5, 6]. Different from radiographic TFOA, the risk factors of the radiographic severity of PFOA have not been as thoroughly studied.

Abnormal PFJ alignment and morphology are hypothesized to alter the mechanics of the PFJ, which can in turn lead to PFOA. PFJ malalignment typically manifests as lateral patellar tilt, lateral displacement, or a combination of the two, which is a deviation of the patella relative to trochlea that may lead to abnormal stress transmitted through the PFJ [7]. A previous study identified the association between PFOA and both abnormal trochlear sulcus morphology and tibiofemoral alignment [8]. However, there is little evidence that patella height, lateral patellar displacement, and patellar tilt are associated with PFOA. A previous study indicated that the vertical position of the patella is associated with patellofemoral malalignment and reduced PFJ contact area in patella Alta [9]. We speculate that the increasing of patella height, sulcus angle (i.e., a flatter trochlear), lateral patellar displacement, and lateral patellar tilt will lead to patella instability and easily cause PFOA. Besides, according to Wiberg's investigations, the patella shape is defined into three types [10]. However, there are few studies about the relationship between patella morphology with the severity of PFOA.

The purpose of this study was to determine the association of PFJ alignment (patella height, lateral patellar displacement, patellar tilt, and lateral patellar angle, TTTG) and PFJ morphologic features (sulcus angle, medial trochlear inclination angle, lateral trochlear inclination angle, trochlear angle, and patella shape) with the severity of PFOA.

\section{Methods}

This study has been approved by the Ethics Committee of our hospital. CT scans and lateral radiograph data of 165 patients with PFOA were analyzed retrospectively between June 2019 and June 2020 at our hospital. All the cases in this study were hospitalized for total knee arthroplasty or arthroscopic surgery. CT scans of the knee joints were performed in the supine position with the knee in extension and lateral radiographs were taken in full weight-bearing holding knees flexed $30^{\circ}$ and feet externally rotated $10^{\circ}$. We staged the severity of PFOA based on Merchant et al. [11]. CT scans were performed to define PFOA due to the PFOA criteria for MRI definition are not well established, and MRI features of PFOA such as PFJ marrow edema and minor cartilage defects are common even in young asymptomatic knees [12]. To be included, participants in this cohort had to have a diagnosis of PFOA confirmed by preoperative CT scan and intraoperative observation, as well as all the cases should have preoperative $\mathrm{CT}$ and lateral radiographs. The exclusion criteria of this study include (1) traumatic
PFOA, (2) patients without CT or lateral radiographs, and (3) previous surgery to the lower extremity. Of the 165 knees analyzed in this study, 15 were excluded due to the inability to identify skeletal landmarks. This occurred because of severe osteoarthritis with severe osteophytes. This left 150 knees (150 patients) eligible for the study. The demographic data of the 150 patients are shown in Table 1.

\section{Assessment of radiographic $\mathrm{OA}$}

Using the CT scan, each knee was evaluated for joint space stenoses of PFJ on a 1-4 stage based on Merchant et al. [11]. Stage 1 is mild, with the joint space of at least $3 \mathrm{~mm}$; stage 2 is moderate, with the joint space less than $3 \mathrm{~mm}$, but no bone contact; stage 3 is severe, with partial bony contact less than $1 / 4$ of the joint surface; and stage 4 is very severe, and the surface of the joint bone is completely touching each other. In this study, we combined stage 3 and stage 4 PFOA into stage 3 . We only assessed the severity of the lateral PFOA, not the medial PFOA, because we did not find a case with isolated medial PFOA through further intraoperative observation. Besides, the severity of TFOA was also evaluated to exclude the interference to PFOA. The radiographic TFOA was evaluated by using the Kellgren and Lawrence grade [13].

\section{Assessment of patellofemoral joint morphology and patellofemoral alignment}

We measured three kinds of morphologic characteristics of the trochlea: the lateral and medial trochlear inclination angle, and sulcus angle (Fig. 1A). The CT image of

\begin{tabular}{ll} 
Table 1 Patients demographics ( $n=150$ knees, 150 patients) \\
\hline Variable (mean \pm SD) & $\boldsymbol{N}(\%)$ \\
\hline Age, years & $60.7 \pm 9.5$ \\
Gender, F & $108(72 \%)$ \\
Side, right & $78(52 \%)$ \\
Sulcus angle & $150.6^{\circ} \pm 9.3^{\circ}$ \\
Lateral trochlear inclination & $16.7^{\circ} \pm 5.2^{\circ}$ \\
Medial trochlear inclination & $12.5^{\circ} \pm 6.7^{\circ}$ \\
Wiberg classification & \\
$\quad$ Type I & $54(36 \%)$ \\
Type II & $75(50 \%)$ \\
Type III & $21(14 \%)$ \\
Congruence angle & $26.4^{\circ} \pm 21.7^{\circ}$ \\
Patella tilt & $15.4^{\circ} \pm 8.3^{\circ}$ \\
Lateral patellar angle & $1.6^{\circ} \pm 6.9^{\circ}$ \\
Caton index & $0.9 \pm 0.1$ \\
TT-TG & $14.9 \mathrm{~mm} \pm 4.8 \mathrm{~mm}$
\end{tabular}

$\pi$-TG tibial tubercle-trochlear groove distance 


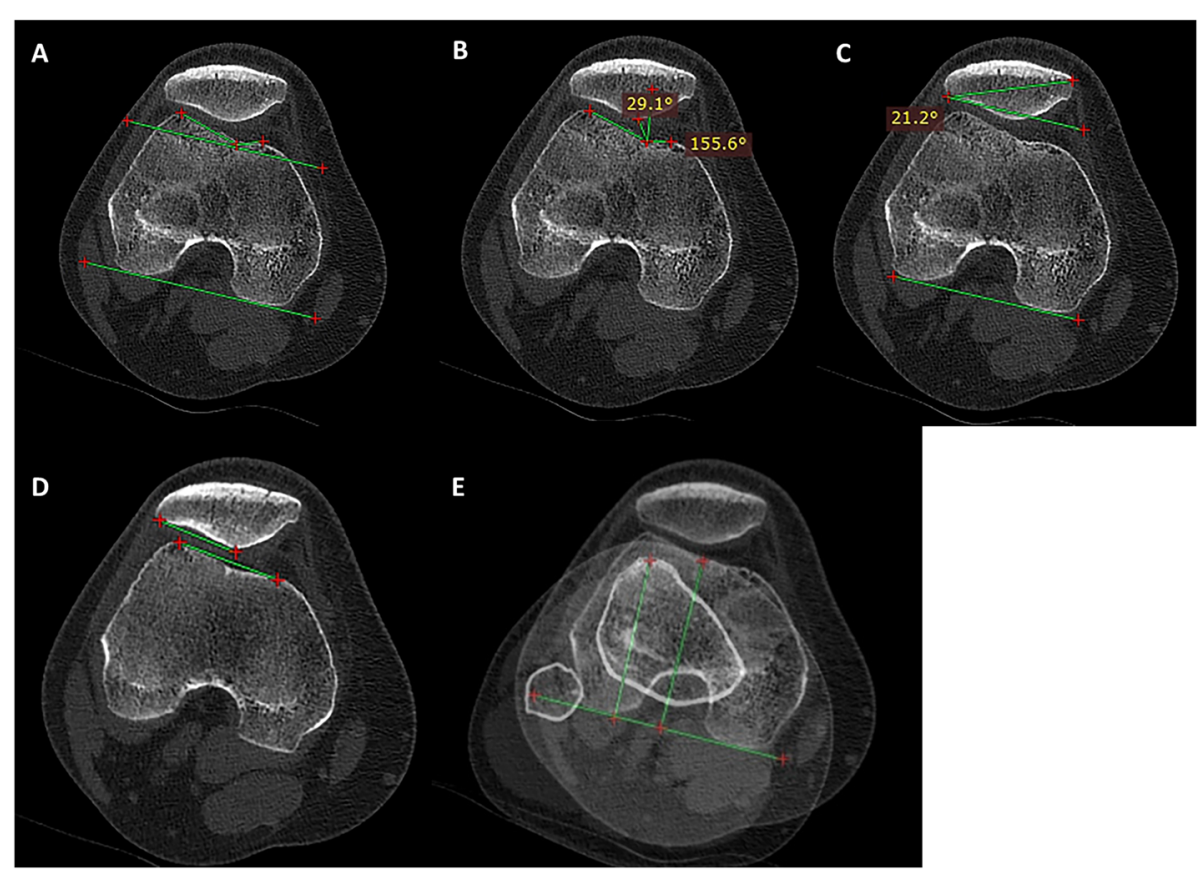

Fig. 1 (A) Measurement of PFJ morphology. (B) Measurement of congruence angle. (C) Measurement of patella tilt. (D) Measurement of lateral patellar angle. (E) Measurement of TT-TG

trochlear morphology was analyzed in the section of the "Roma Dome." The morphology of the patella is classified by Wiberg classification according to CT images [10]. In type I, the medial and lateral facets are symmetrical, and of equal size. In type II, the medial facet is smaller and flatter than the lateral facet. In type III, the medial facet is convex, more vertical, and significantly smaller.

We measured five different aspects of the patellofemoral alignment: The congruence angle (Fig. 1B), patellar tilt (Fig. 1C), and lateral patellar angles (Fig. 1D); patella height (i.e., Caton-Deschamps index) (Fig. 2) and TT-TG (Fig. 1E). Morphologic features of the trochlea, lateral displacement (the congruence angle), patellar tilt, and lateral patellar angles were measured on a CT scan [14]. Patella height (Caton index) [15] was measured on the full weight-bearing lateral radiographs. TT-TG was measured by two scanning slices, and the method with a technique was described by Schoettle et al. [16]. Specific methods are depicted in Table 2.

\section{Statistical analysis}

All statistics were analyzed by the SPSS software (IBM, Armonk, NY, USA, v. 24.0). We randomly selected 30 knees to test the intra- and interobserver reliability. Two experienced surgeons using the same standard criteria independently measured the data twice with an interval of 4 weeks and then calculated the intra- and interclass correlation coefficient (ICC). After a normal distribution was ensured, the relationship between all the measures of patellofemoral morphology and alignment with the radiographic severity of lateral PFOA was explored by using conditional multivariable logistic regression (the grading III as the referent category). To avoid the effect of the degree of TFOA on the outcome, radiographic TFOA was adjusted in the multivariable regression model. A power analysis was undertaken. At least 138 cases were needed to reach an effect size of 0.15 , a power of 0.95, and an alpha of 0.05 using G*Power v. 3.1 (G* Power, Dusseldorf, Germany). Statistical significance was set at $p<0.05$.

\section{Results}

At last, a total of 150 knees (150 patients) were included in this study, including 108 females (72\%) and 42 males (28\%), 78 (52\%) right knees, and $72(48 \%)$ left knees. The mean age was 60.7 years $(\mathrm{SD}=9.5)($ Table 1$)$. The results characterizing the patellofemoral morphology and alignment are shown in Table 1 . The ICC results ranged from 0.838 to 0.969 , which indicated excellent agreement (Table 3).

The relationship between patellofemoral alignment and morphology with the radiographic severity of lateral PFOA (the grading 3 as the referent category) is shown in Table 4. The sulcus angle and lateral patellar angle were negatively correlated with the radiographic severity 


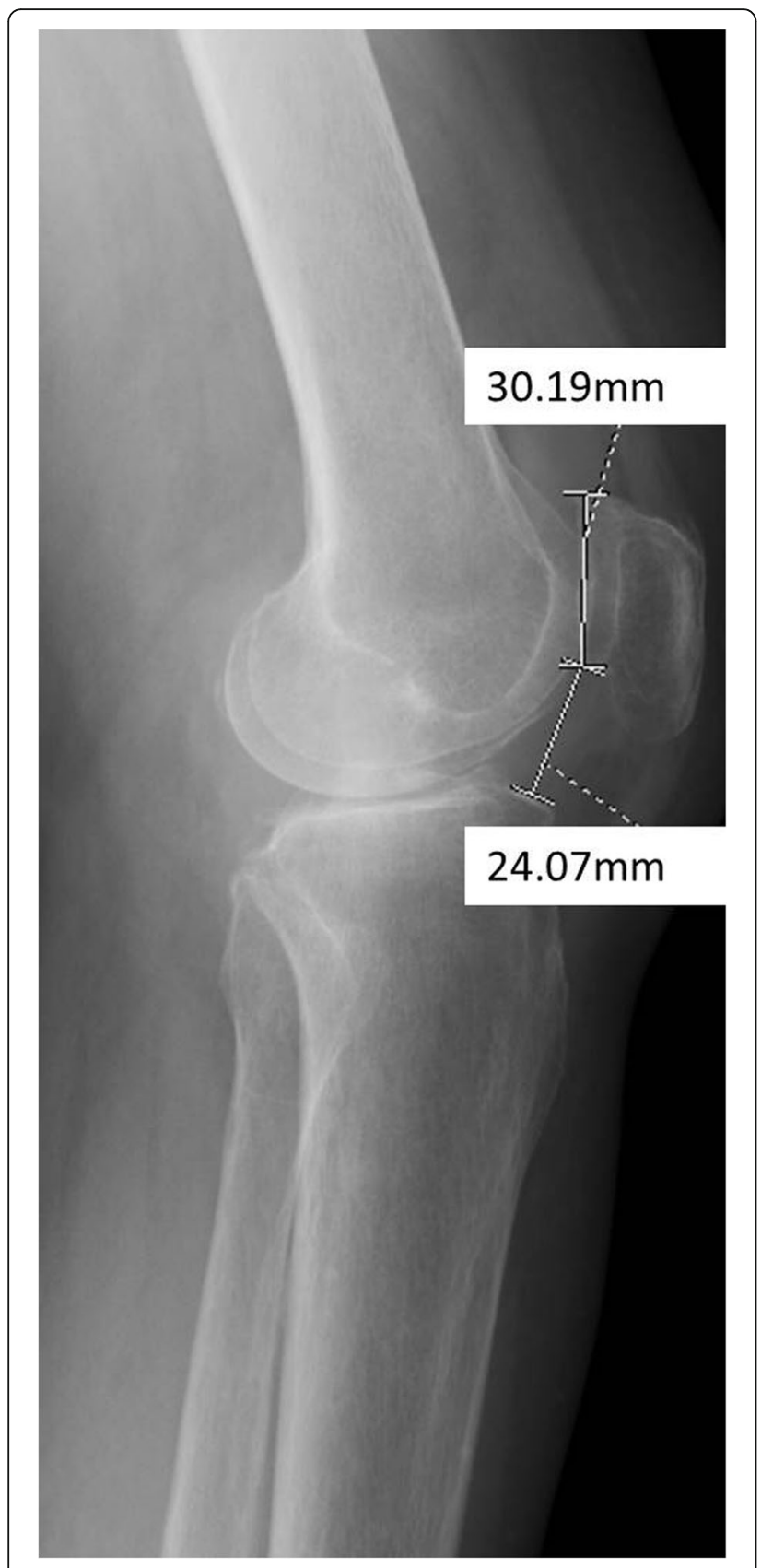

Fig. 2 Measurement of patella height (Caton-Deschamps index)

of lateral PFOA, while the congruence angle and patella morphology were positively correlated with the radiographic severity of lateral PFOA. More notably, a statistically U-shaped relationship was observed between the patellar height and severity of lateral PFOA, which indicated that the highest or lowest quarter of patella height has a higher incidence of more severe lateral PFOA than the middle two-quarters of the patellar height $(p=$ 0.011 ).

When compared with the highest quarter of the sulcus angle and lateral patellar angle, the adjusted ORs of the radiographic grade 3 of lateral PFOA were 8.8 for the lowest quarter of the sulcus angle $(P=0.043)$ and 16.51 for the lowest quarter of the lateral patellar angle $(P<$ $0.001)$, respectively. Similarly, when compared with the highest quarter of the congruence angle and type III patellar morphology, the adjusted ORs of the radiographic grade 3 of lateral PFOA were 0.04 for the lowest quarter of the congruence angle $(P<0.001)$ and 0.18 for the type I patellar morphology $(P=0.048)$, respectively (Table 4$)$. Besides, when radiographic TFOA is adjusted, there is no change in the results (adjusted OR) substantially.

\section{Discussion}

In this study, we evaluated various measures of PFJ morphology and alignment to determine which could predict the radiographic severity of lateral PFOA. The most important findings of the present study were as follows: (1) deeper trochlea (smaller sulcus angle), patellar lateral displacement (larger congruence angle), and patellar lateral tilt (smaller lateral patellar angle) are associated with more severe lateral PFOA; (2) type III patellar morphology is associated with more severe lateral PFOA; and (3) a statistically U-shaped relationship between the patellar height (Caton-Deschamps index) and severity of lateral PFOA.

Previous studies identified that most of the variability in alignment can be explained by the combination of morphology parameters, highlighting the complex interaction between patellofemoral joint morphology and alignment [17, 18]. These intricate interactions between morphology and alignment may result in pathologic alterations of the patellofemoral joint. In most patients, such a multifaceted etiology makes the identification of a single pathological factor for PFOA extremely challenging. Therefore, we evaluated PFJ morphology and alignment to determine which predicted the radiographic severity of lateral PFOA. All the cases in this study were hospitalized for total knee arthroplasty or arthroscopic surgery. Through further intraoperative observation, we did not find a case with isolated medial PFOA, possibly because the medial patellar instability is an objective iatrogenic condition [19]. Therefore, we just analyzed the influencing factors for the severity of lateral PFOA in all cases. In this study, we identified a statistically U-shaped relationship between the patellar height (CatonDeschamps index) and severity of lateral PFOA, which suggested that extreme values of the patellar height have a higher incidence of more severe lateral PFOA. Several previous studies suggested the patella Alta as a possible risk factor for PFOA [20, 21], while some other studies suggested that patella Baja can produce increased patellofemoral contact pressures at early knee flexion, which can potentiate arthritis progression [22, 23]. We propose several explanations for this finding. On the one hand, a 
Table 2 Measurement methods of the parameters

\begin{tabular}{|c|c|c|}
\hline Parameters & & Definition \\
\hline \multicolumn{3}{|l|}{ PFJ morphology } \\
\hline Sulcus angle & SA & $\begin{array}{l}\text { This angle is defined by lines joining the highest points of the medial and lateral condyles and the lowest } \\
\text { point of the intercondylar sulcus }\end{array}$ \\
\hline Lateral trochlear inclination & LTI & The angle between the lateral facet line of trochlea and posterior condylar line \\
\hline Medial trochlear inclination & MTI & The angle between the medial facet line of trochlea and posterior condylar line \\
\hline Wiberg classification & Wiberg & $\begin{array}{l}\text { Type I, the facets are symmetrical, concave, and of equal size } \\
\text { Type II, the medial facet is smaller and flatter compared to the lateral facet } \\
\text { Type III, the medial facet is convex, more vertical, and markedly smaller }\end{array}$ \\
\hline \multicolumn{3}{|l|}{ PFJ alignment } \\
\hline Congruence angle & CA & The line through the apex of the patella to a line bisecting the trochlea \\
\hline Patella tilt & PT & The angle between the posterior condylar line and the maximal patella width line \\
\hline Lateral patellar angle & LPA & $\begin{array}{l}\text { The angle between the lateral facet line of patella and the line joining the highest points of the medial and } \\
\text { lateral condyles }\end{array}$ \\
\hline Caton-Deschamps index & $\mathrm{CDI}$ & $\begin{array}{l}\text { The ratio between the length of the distance from the lower edge of the patellar articular surface to the } \\
\text { anterior superior surface of the tibial plateau and the length of the patellar articular surface }\end{array}$ \\
\hline $\begin{array}{l}\text { Tibial tubercle-trochlear } \\
\text { groove distance }\end{array}$ & TT-TG & $\begin{array}{l}\text { The TT-TG distance is the distance from the deepest point of the trochlear groove to the highest point of } \\
\text { the tibial tuberosity }\end{array}$ \\
\hline
\end{tabular}

PFJ patellofemoral joint

higher sagittal position of the patella would lead to a reduced PFJ contact area during knee extension. These abnormal mechanical mechanics of PFJ can lead to patellar instability, PFJ malalignment, and increased shear forces and PFJ contact pressure, which may be risk factors for PFOA [24, 25]. On the other hand, a lower patella may create excessive stress on the articular surface at knee flexion, which causes degeneration of chondrocytes and contributes to the formation of PFOA.

Furthermore, our study also suggested that a smaller sulcus angle (a narrow trochlea) increased the severity of lateral PFOA, but not flatter trochlea. This is different from our speculation that greater sulcus angle increased prevalent PFOA, which was identified in some previous studies [26, 27]. Ali et al. indicated that a larger sulcus angle was associated with severe cartilage defects [28]. Similarly, another study indicated an association between larger sulcus angle and PFJ cartilage injury [27]. However, our study identified a different view that narrow trochlea increased prevalent PFOA. We hypothesized that a narrow trochlea may produce a smaller contact area in PFJ, resulting in excessive stress on the PFJ surface. Meanwhile, shallower trochlear sulcus could increase the PFJ contact area and reduce the articular surface contact pressure, which produces a better distribution of the stress on PFJ.

The association of the patella morphology and PFOA has only been sparsely reported in the

Table 3 Results of radiological parameters of PFJ morphology and alignment and interclass correlation coefficient tests

\begin{tabular}{|c|c|c|c|}
\hline Parameters & Mean (SD; range) & Interobserver ICC (95\% CI) & Intraobserver ICC (95\% Cl) \\
\hline Joint space narrowing & $1.9 \mathrm{~mm}(1.1 \mathrm{~mm} ; 0 \mathrm{~mm}$ to $4.47 \mathrm{~mm})$ & 0.956 (0.906 to 0.979$)$ & 0.962 (0.920 to 0.982 ) \\
\hline \multicolumn{4}{|l|}{ PFJ morphology } \\
\hline Sulcus angle & $150.6^{\circ}\left(9.3^{\circ} ; 124^{\circ}\right.$ to $\left.173^{\circ}\right)$ & 0.878 (0.703 to 0.939$)$ & 0.906 (0.800 to 0.956$)$ \\
\hline Lateral trochlear inclination & $16.7^{\circ}\left(5.2^{\circ} ; 4^{\circ}\right.$ to $\left.29^{\circ}\right)$ & $0.852(0.684$ to 0.930$)$ & 0.948 (0.876 to 0.941$)$ \\
\hline Medial trochlear inclination & $12.5^{\circ}\left(6.7^{\circ} ; 1^{\circ}\right.$ to $\left.36^{\circ}\right)$ & 0.845 (0.651 to 0.947$)$ & 0.939 (0.870 to 0.971$)$ \\
\hline Wiberg classification & - & 0.865 (0.712 to 0.937$)$ & 0.869 (0.721 to 0.938$)$ \\
\hline \multicolumn{4}{|l|}{ PFJ alignment } \\
\hline Congruence angle & $26.4^{\circ}\left(21.7^{\circ} ;-38\right.$ to 74$)$ & 0.860 (0.678 to 0.921$)$ & 0.964 (0.922 to 0.983$)$ \\
\hline Patella tilt & $15.4^{\circ}\left(8.3^{\circ} ; 0\right.$ to 43$)$ & 0.969 (0.934 to 0.985$)$ & 0.935 (0.863 to 970$)$ \\
\hline Lateral patellar angle & $1.63^{\circ}\left(6.9^{\circ} ;-28^{\circ}\right.$ to $\left.16^{\circ}\right)$ & 0.915 (0.781 to 0.912$)$ & 0.913 (0.815 to 959) \\
\hline Caton-Deschamps index & $0.9(0.1 ; 0.60$ to 1.34$)$ & 0.838 (0.655 to 0.924$)$ & 0.914 (0.816 to 0.960$)$ \\
\hline TT-TG & $14.9(4.8 ; 0.0$ to 28.9$)$ & 0.919 (0.827 to 0.962$)$ & 0.974 (0.945 to 0.988$)$ \\
\hline
\end{tabular}

PFJ patellofemoral joint, $\pi$-TG tibial tubercle-trochlear groove distance 
Table 4 Relationship between patellofemoral alignment and morphology with the radiographic severity of lateral PFOA (the grading 3 of PFOA as the referent category)

\begin{tabular}{|c|c|c|c|c|c|}
\hline Measures & Quarter & Range & $N$ of cases & OR & Adjusted OR \\
\hline \multirow[t]{4}{*}{ SA (degree) } & 1 & $124.0-142.0$ & 44 & 7.66 & 8.80 \\
\hline & 2 & $142.1-150.0$ & 36 & 5.14 & 6.00 \\
\hline & 3 & $150.1-157.3$ & 38 & 1.57 & 1.37 \\
\hline & 4 & $157.4-173.0$ & 32 & 1.00 & 1.00 \\
\hline \multirow[t]{3}{*}{ Wiberg classification } & । & - & 54 & 0.23 & 0.18 \\
\hline & $\|$ & - & 75 & 0.48 & 0.39 \\
\hline & III & - & 21 & 1.00 & 1.00 \\
\hline \multirow[t]{4}{*}{ CA (degree) } & 1 & $-38.0-9.0$ & 37 & 0.06 & 0.04 \\
\hline & 2 & $9.1-18.5$ & 43 & 0.25 & 0.22 \\
\hline & 3 & 18.6-33.8 & 35 & 0.47 & 0.43 \\
\hline & 4 & $33.9-58.0$ & 35 & 1.00 & 1.00 \\
\hline \multirow[t]{4}{*}{ CDI } & 1 & $0.60-0.78$ & 35 & 1.75 & 1.21 \\
\hline & 2 & $0.79-0.91$ & 39 & 0.84 & 0.70 \\
\hline & 3 & $0.91-1.03$ & 36 & 0.72 & 0.52 \\
\hline & 4 & $1.03-1.39$ & 40 & 1.00 & 1.00 \\
\hline \multirow[t]{4}{*}{ LPA (degree) } & 1 & $-15.0-1.0$ & 38 & 17.55 & 16.51 \\
\hline & 2 & $1.1-4.0$ & 37 & 2.54 & 2.52 \\
\hline & 3 & $4.1-7.0$ & 33 & 2.12 & 2.08 \\
\hline & 4 & 7.1-16.0 & 42 & 1.00 & 1.00 \\
\hline
\end{tabular}

SA sulcus angle, $C A$ congruence angle, $L P A$ lateral patellar angle, $C D I$ Caton-Deschamps index

literature. In our patient population, the patella type, classified according to Wiberg, showed a significant prevalence of type II patella (74\%), followed by type I patella (36\%) and type III patella (14\%). In this study, type III patellar morphology is associated with more severe lateral PFOA. A previous study showed that Wiberg patella type III, which presented a medial border dysplasia or a short patellar apex, is more often involved in patients with patellar dislocation [29], and patella lateral displacement is associated with more severe lateral PFOA [30]. On the other hand, Wiberg type III patella presented only a small amount of contact with the medial femoral condyle due to the low surface area of the medial facet, which increased the contact pressure of the lateral patella facet on the lateral femoral condyle during knee flexion.

Besides, we also identified that congruence angle was associated with the severity of lateral PFOA positively, while lateral patellar angle was associated with the severity of lateral PFOA negatively. These results are consistent with the results of previous studies that patella lateral displacement or patella tilt could reduce the contact area of the PFJ and increased contact stress on the PFJ surface [31, 32]. Excessive stress on the lateral articular surface of the PFJ could increase the risk of lateral PFOA. This may be why previous studies recommended applying a knee taping to force the patella medially and away from the overloaded lateral compartment as a treatment for PFOA [33, 34].

Several strengths of our study are remarkable. First, the CT scan of the knee was used to define the radiographic PFOA rather than the skyline view of knee radiographs. CT image facilitates the evaluation of radiographic PFOA and measurement morphology and alignment of PFJ. Second, it is worth mentioning that there were no substantial changes in the results when adjusting for radiographic TFOA, suggesting that our findings were not confused by radiographic TFOA.

We recognize several limitations in this study. Firstly, CT was used to define the PFOA. CT is not as sensitive as MRI in the detection of structural lesions. Nevertheless, all patients in this study were hospitalized for total knee arthroplasty or arthroscopic surgery, so our findings by CT were consistent with the results of further intraoperative observation, which suggested the robustness of our findings. Secondly, the patella alignment was measured by CT scans during knee extension. Therefore, the influence of the patella and femoral movement during knee flexion cannot be considered when measuring patella alignment. In the future, it is necessary to measure and analyze the PFJ alignment at different flexion 
angles. Thirdly, our study only analyzed the imaging findings, which need to be studied in combination with the patient's symptoms in the future.

\section{Conclusions}

In conclusion, our results identified that there was a Ushaped association between the patellar height and the severity of lateral PFOA. Moreover, patella lateral tilt, lateral displacement, and type III patella are also associated with the severity of lateral PFOA. This study is of clinical significance because identifying the role of PFJ malalignment and abnormal morphology in the severity of PFOA could provide early intervention strategies for patients who may increase the risk of developing PFOA.

\section{Abbreviations}

PFOA: Patellofemoral osteoarthritis; PFJ: Patellofemoral joint; TTT: Tibial tubercle-trochlear groove distance; TFOA: Tibiofemoral osteoarthritis

\section{Acknowledgements}

The authors would like to thank all the staff of the participating departments.

\section{Authors' contributions}

NCD and AG designed the study. YKD and HYY performed the experimental work. HRZ and CYX evaluated the data. YKD wrote the manuscript. All authors read and approved the final manuscript.

\section{Funding}

Not applicable

\section{Availability of data and materials}

The detailed data and materials of this study are available from the corresponding author via e-mail on reasonable request.

\section{Declarations}

\section{Ethics approval and consent to participate}

This study was inspected and approved by the ethics committee of Beijing Friendship Hospital.

\section{Consent for publication}

Not applicable.

\section{Competing interests}

All authors declare that they have no competing interests.

Received: 22 June 2021 Accepted: 19 August 2021

Published online: 04 September 2021

\section{References}

1. Kobayashi S, Pappas E, Fransen M, Refshauge K, Simic M. The prevalence of patellofemoral osteoarthritis: a systematic review and meta-analysis. Osteoarthritis Cartilage. 2016;24(10):1697-707. https://doi.org/10.1016/j.joca.2 016.05.011.

2. Duncan R, Hay E, Saklatvala J, Croft P. Prevalence of radiographic osteoarthritis: it all depends on your point of view. Rheumatology. 2006; 45(6):757-60. https://doi.org/10.1093/rheumatology/kei270.

3. Iwano T, Kurosawa H, Tokuyama H, Hoshikawa Y. Roentgenographic and clinical findings of patellofemoral osteoarthrosis: with special reference to its relationship to femorotibial osteoarthrosis and etiologic factors. Clin Orthop Relat Res. 1990:252:190-7.

4. Casscells SW. Gross pathological changes in the knee joint of the aged individual: a study of 300 cases. Clin Orthop Relat Res. 1978;132:225-32.

5. McAlindon T, Zhang Y, Hannan M, Naimark A, Weissman B, Castelli W, et al. Are risk factors for patellofemoral and tibiofemoral knee osteoarthritis different? J Rheumatol. 1996;23(2):332-7.
6. Cooper C, McAlindon T, Snow S, Vines K, Young P, Kirwan J, et al. Mechanical and constitutional risk factors for symptomatic knee osteoarthritis: differences between medial tibiofemoral and patellofemoral disease. J Rheumatol. 1994:21(2):307-13.

7. Grelsamer RP, Weinstein CH. Applied biomechanics of the patella [Review] [39 refs]. Clin Orthop. 2001;389:9-14. https://doi.org/10.1097/00003086-2001 08000-00003.

8. Macri EM, Stefanik JJ, Khan KK, et al. Is Tibiofemoral or patellofemoral alignment or trochlear morphology associated with patellofemoral osteoarthritis? A systematic review. Arthritis Care Res (Hoboken). 2016;68: 1453-70.

9. Ward SR, Terk MR, Powers CM. Patella alta: association with patellofemoral alignment and changes in contact area during weight-bearing. J Bone Joint Surg Am. 2007;89(8):1749-55. https://doi.org/10.2106/JBJS.F.00508.

10. Wiberg G. Roentgenographic and anatomic studies on the femoropatellar joint. Acta Orthop Scand. 1941;12:319-410.

11. Merchant $A C$, Mercer $R L$, Jacobsen $R G$, Cool CR. Roentgenographic analysis of patellofemoral congruence. J Bone J Surg Am. 1974;56A:1391.

12. van der Heijden RA, de Kanter JL, Bierma-Zeinstra SM, Verhaar JA, van Veldhoven PL, Krestin GP, et al. Structural abnormalities on magnetic resonance imaging in patients with patellofemoral pain a cross-sectional case-control study. Am J Sport Med. 2016;44(9):2339-46. https://doi.org/1 $0.1177 / 0363546516646107$

13. Kohn MD, Sassoon AA, Fernando ND. Classifications in Brief: KellgrenLawrence Classification of Osteoarthritis. Clin Orthop Relat Res. 2016;474(8): 1886-93. https://doi.org/10.1007/s11999-016-4732-4.

14. Inoue M, Shino K, Hirose H, Horibe S, Ono K. Subluxation of the patella. Computed tomography analysis of patellofemoral congruence. J Bone Joint Surg Am. 1988;70(9):1331-7. https://doi.org/10.2106/00004623-19887009000009.

15. Caton J, Deschamps G, Chambat P, Lerat JL, Dejour H. Patella infera: a propos of 128 cases. Rev Chir Orthop Reparatrice Appar Mot. 1982;68(5): 317-25.

16. Schoettle PB, Zanetti M, Seifert B, Pfirrmann CWA, Fucentese SF, Romero J. The tibial tuberosity-trochlear groove distance; a comparative study between CT and MRI scanning. Knee. 2006;13(1):26-31. https://doi.org/10.1 016/j.knee.2005.06.003

17. Harbaugh CM, Wilson NA, Sheehan FT. Correlating femoral shape with patellar kinematics in patients with patellofemoral pain. J Orthop Res. 2010; 28(7):865-72. https://doi.org/10.1002/jor.21101.

18. Teng HL, Chen YJ, Powers CM. Predictors of patellar alignment during weight bearing: an examination of patellar height and trochlear geometry. Knee. 2014;21(1):142-6. https://doi.org/10.1016/j.knee.2013.08.011.

19. Sanchis-Alfonso V. How to deal with chronic patellar instability: what does the literature tell us? Sports Health. 2016;8(1):86-90. https://doi.org/10.11 77/1941738115604156.

20. Stefanik JJ, Guermazi A, Zhu Y, Zumwalt AC, Gross KD, Clancy M, et al. Quadriceps weakness, patella alta, and structural features of patellofemoral osteoarthritis. Arthritis Care Res (Hoboken). 2011;63(10):1391-7. https://doi. org/10.1002/acr.20528.

21. Stefanik JJ, Zhu Y, Zumwalt AC, Gross KD, Clancy M, Lynch JA, et al. Association between patella alta and the prevalence and worsening of structural features of patellofemoral joint osteoarthritis: the multicenter osteoarthritis study. Arthritis Care Res (Hoboken). 2010;62(9):1258-65. https://doi.org/10.1002/acr.20214.

22. Luyckx T, Didden $\mathrm{K}$, Vandenneucker $\mathrm{H}$, et al. Is there a biomechanical explanation for anterior knee pain in patients with patella alta? Influence of patellar height on patellofemoral contact force, contact area and contact pressure. J Bone Joint Surg Br. 2009;91:344-50.

23. Fernandez JW, Akbarshahi M, Crossley KM, Shelburne KB, Pandy MG. Model predictions of increased knee joint loading in regions of thinner articular cartilage after patellar tendon adhesion. J Orthop Res. 2011;29(8):1168-77. https://doi.org/10.1002/jor.21345

24. Ward SR, Powers CM. The influence of patella alta on patellofemoral joint stress during normal and fast walking. Clin Biomech (Bristol, Avon). 2004, 19 1040-7.

25. Ward SR, Terk MR, Powers CM. Patella alta: association with patellofemoral alignment and changes in contact area during weight-bearing. J Bone Joint Surg Am. 2007;89(8):1749-55. https://doi.org/10.2106/JBJS.F.00508.

26. Kalichman L, Zhang Y, Niu J, Goggins J, Gale D, Felson DT, et al. The association between patellar alignment and patellofemoral joint 
osteoarthritis features—an MRI study. Rheumatology. 2007;46(8):1303-8. https://doi.org/10.1093/rheumatology/kem095.

27. Stefanik JJ, Roemer FW, Zumwalt AC, Zhu Y, Gross KD, Lynch JA, et al. Association between measures of trochlear morphology and structural features of patellofemoral joint osteoarthritis on MRI: the MOST study. J Orthop Res. 2012;30(1):1-8. https://doi.org/10.1002/jor.21486.

28. Ali SA, Helmer R, Terk MR. Analysis of the patellofemoral region on MRl: association of abnormal trochlear morphology with severe cartilage defects. AJR Am J Roentgenol. 2010;194(3):721-7. https://doi.org/10.2214/AJR.09.3 008.

29. Servien E, Ait Si Selmi T, Neyret P. Study of the patellar apex in objective patellar dislocation. Rev Chir Orthop Reparatrice Appar Mot. 2003;89(7):60512.

30. Zhao y C, Gao z X, Liu x Q, Li x k Z, Qiu x Y, Li R, et al. Associations of trochlea morphology and patellofemoral alignment with prevalent radiographic patellofemoral osteoarthritis. Osteoarthritis and Cartilage. 2020; 28:824-30.

31. Farrokhi S, Keyak JH, Powers CM. Individuals with patellofemoral pain exhibit greater patellofemoral joint stress: a finite element analysis study. Osteoarthr Cartil. 2011;19(3):287-94. https://doi.org/10.1016/j.joca.2010.12. 001.

32. Stephen JM, Kader D, Lumpaopong P, Deehan DJ, Amis AA. Sectioning the medial patellofemoral ligament alters patellofemoral joint kinematics and contact mechanics. J Orthop Res. 2013;31(9):1423-9. https://doi.org/10.1002/ jor.22371.

33. Hinman RS, Crossley KM, McConnell J, Bennell KL. Efficacy of knee tape in the management of osteoarthritis of the knee: blinded randomized controlled trial [see comment]. BMJ. 2003;327(7407):135-0. https://doi.org/1 0.1136/bmj.327.7407.135

34. Cushnaghan J, McCarthy C, Dieppe P. Taping the patella medially: a new treatment for osteoarthritis of the knee joint? BMJ. 1994;308(6931):753-5. https://doi.org/10.1136/bmj.308.6931.753.

\section{Publisher's Note}

Springer Nature remains neutral with regard to jurisdictional claims in published maps and institutional affiliations.

Ready to submit your research? Choose BMC and benefit from:

- fast, convenient online submission

- thorough peer review by experienced researchers in your field

- rapid publication on acceptance

- support for research data, including large and complex data types

- gold Open Access which fosters wider collaboration and increased citations

- maximum visibility for your research: over $100 \mathrm{M}$ website views per year

At $\mathrm{BMC}$, research is always in progress.

Learn more biomedcentral.com/submissions 